\title{
Blockchain Adoption: Applications and Challenges
}

\author{
M.O. Momoh ${ }^{1}$, P.U. Chinedu², W. Nwankwo ${ }^{3}$, D. Aliu² and M.S. Shaba ${ }^{4}$ \\ ${ }^{1}$ Faculty of Air Engineering, Air Force Institute of Technology Kaduna, Nigeria. \\ 2Department of Computer Engineering, Edo University lyamho, Edo State, Nigeria \\ ${ }^{3}$ Department of Computer Science, Edo University lyamho, Edo State, Nigeria \\ ${ }^{4}$ Federal College of Freshwater Fisheries Technology, New Bussa, Nigeria
}

\begin{abstract}
In recent times, more scholastic and social attention have been paid to blockchain and its distributed ledger system mechanism. The reasons for this ever-increasing attention cannot be far-fetched: blockchain now occupies a copious position in the present-day ways of doing things economically, digitally and 'digital-socially'. Blockchain could be described as a distributed ledger system that allows secure transactions without a central management system. In this distributed ledger system, transactions are coded into blocks, which are linked to each other in the form of a chain. The first application of blockchain is in the bitcoin cryptocurrency. Though not limited to bitcoin, blockchain finds usefulness in security and trusts for instance, digital assets could be coded into blocks to ensure and enforce quality of trust. Consequent upon the quality of trust the blockchain confers on a digital asset, transparency among participating nodes is guaranteed. This is because, any change made to any record in a given block automatically initiates and enforces a corresponding change in all other blocks in the chain hence tampering or breach is almost impossible. Owing to its impressive prospects in the socioeconomic and political ecosystem, this paper was conceived to examine the current developments around this novel technology with particular emphasis on its benefits and proposed challenges and needs to fill the gap created in the vital socioeconomic domains. The paper concludes that the blockchain technology is a plausible approach to restoring the trust, confidentiality, availability and integrity in transactions in the cyberspace and the world at large as majority of the global economy thrives in the cloud.
\end{abstract}

ARTICLE HISTORY

Received: 14 June 2021

Revised: 22 July 2021

Accepted: 24 August 2021

\section{KEYWORDS}

Blockchain

Bitcoin

Distributed Ledger System

Hash

Data Structure

\section{INTRODUCTION}

Blockchain can be described as a chain data structure that allows an open and fairness transaction among participating nodes using the principle of distributed digital ledger technology [1]. The distributed digital ledger system ensures integrity, transparency and auditability of records in the blockchain. Records in the blockchain are tamperproof, i.e. cannot be easily tampered without automatic notification transmitted to other nodes [2]. Blockchain technology encompasses several other technologies put forward together to address the issue of double spending, trust and security of transactions. Key among the technologies are: distributed (shared) ledger, peer to peer transmission, consensus mechanism, smart contract and encryption algorithm [3]. Blockchain system has no central authority where the flow of transactions is managed; thus, all nodes in the chain have equal capability. The computers that make up the network are in more than one location.

Blockchain is akin to a public ledger that stores information in a chain of blocks. Blockchain systems allows a group of connected computers to maintain a uniform account together without a third-party intervention. Each node in the network is like a database, it keeps record of all other nodes [4]. Blockchain is mainly used for storing information, it can also be referred to as a database system because information can be written into or read from the blockchain [5]. Blockchain has a large distribution of independent nodes. An individual can quickly join the blockchain network by setting up a server, thus becoming a node. The absence of a central node in the blockchain system gives the nodes equal capability. To ensure consistency in the blockchain, all nodes are synchronized and updated when a permissioned node joins the chain. In this paper, we presented some identified benefits and challenges of blockchain technology. More so, characteristics and key technologies that are inherent in the blockchain technology are discussed. The remainder of the paper is organized as follows: Section II present some related work. Section III described the blockchain formation process, the key technology of blockchain is presented in Section IV while section V presents the key characteristics. Section VI and VII presents the applications and challenges respectively and section VIII concludes the paper.

\section{RELATED WORK}

This section provides some review on Blockchain technology, its efficiency, applications domain and security concern. The idea of Blockchain technology was birthed to solve the problem of double spending with the aid of asymetric 
cryptography, where by each participating node is assigned a private key (for decryption) and public key is shared with all other nodes[6]. Transaction in the chain are coded into blocks, each and every of this transaction is verified by the majority of the participants in the system, thus, making fradulent trasactions nearly impossible [6]. Existing research on blockchain has been majorly on the efficiency of the technology, applications and security issues.

Efficiency is one of the greatest challenges in the design domain of blockchain. It takes a rigorous verifcation process in the blockchain in other to create a new transaction of records, these leads to waste of computing resources and latency in the confirmation time[6-7]. Currently, it takes about 10 minutes for a transaction in a blockchain to be confirmed. More so, thousands on nodes are competing to verify transactions, these also limit the the applications domain of blockchain. For instance, the current blockchain techniques are practically not suitable for IoT networks because IoT devices are designed to work on low power and low computation capability [7]. In other to improve the efficiency of blockchain, [8] proposed a lightweight blockchain arhitecture for protection of personal data. In their work, they improve the efficiency of the blockchain by using off-chain data storage and heavy processing. However, only lightweight processing and reference to data were managed in the blockchain. A scheme that could lead to energy efficient in Bitcoin was proposed by [9]. They amended the extra bytes to the present block header so as to utilize the the timestamp more efficiently.

Several work has been done on the applications domain of blockchain technology. A research was carried out on the application of blockchain in the traceability system of agricultural product by [10], the authors proposed a traceability system model based on the technology of blockchain for agricultural product. Similarly, a traceability system model for mineral and metal supply chain was also prosed by [11]. More so, [5] carried out a surevy on the application of blockchain technology in the auditing environment. A systematic review was also documented by [12] on the benefits of blockchain in cloud computing.

It was documented in [13] that the early adopters of blockchain in the finance sector considered it worthwhile because of its unique immutability characteristics and other features to secure transactions. However, blockchain has been found to be vulnerable to $51 \%$ attacks. The $51 \%$ attack is exclusive to blockchain system and it occur when a node controls a minimum of $51 \%$ processing power of the blockchain. More so, according to the analysis by [14], they found out that there are still security breaches that occurred, such as Distributed Denial of Service (DDOS) attacks and private accont hacking. Howevre, to fix the various security breaches and anonymity flaws in blockchain, much work has been done and many schemes has been proposed by several researchers [15-17].

\section{BLOCKCHAIN FORMATION PROCESS}

Blockchain are made up of these entities- block, and chain. A block bears a somewhat resemblance to a database record, and the essence of writing a data is to form a block. Every block is made up of both block head and block body. The containments of every block head are the eigenvalues of the current block i.e. the hash of the actual data, hash of the previous block and the generation time etc. In order to prevent the ease of changing the vital contents of the previous block, hashing is used. An effective hashing function should have these 3 properties; collision resistance (there should be difficulty in having 2 different messages with same hash value), pre-image resistance (for a given hash value, there should not be ease in accessing a message with the same hash value) and second pre-image resistance (for a particular message, there should be rigour in having another message with same hash value). A modification of the content of the current block or hash of the last block will initiate a corresponding modification of the hash of the current block. The worthiness of this cannot be undermined; changing a block implies changing the hash of the block. In order to modify a block, the person must change all the blocks in the chain (because the next block contains the hash of the previous block), otherwise the blocks that have been removed will be cut off from the blockchain. It is nearly impossible to modify a number of blocks in a short time, unless someone has mastered the computing power (at least 51\%) of the entire network, thus, hash's calculation is very laborious. Reliability of a blockchain is guaranteed through this tandem arrangement of blocks, and once the data is written, it cannot be tampered. This is like historic events, what happened was happening, and never changed [10]. The connection of each block to the previous block to form a chain is the origin of the name "Blockchain". During the formation of blockchain, a bifurcation is formed when two nodes write data to the blockchain simultaneously. The new node always joined the longest blockchain, if the blockchain has a fork, the node will automatically joined the chain with the highest computing power [10].

\section{THE KEY TECHNOLOGY OF BLOCKCHAIN}

Blockchain technology comprises several technologies that are integrated to address the issue of trust and security in transaction. Figure 1 shows the four key technologies of blockchain. 


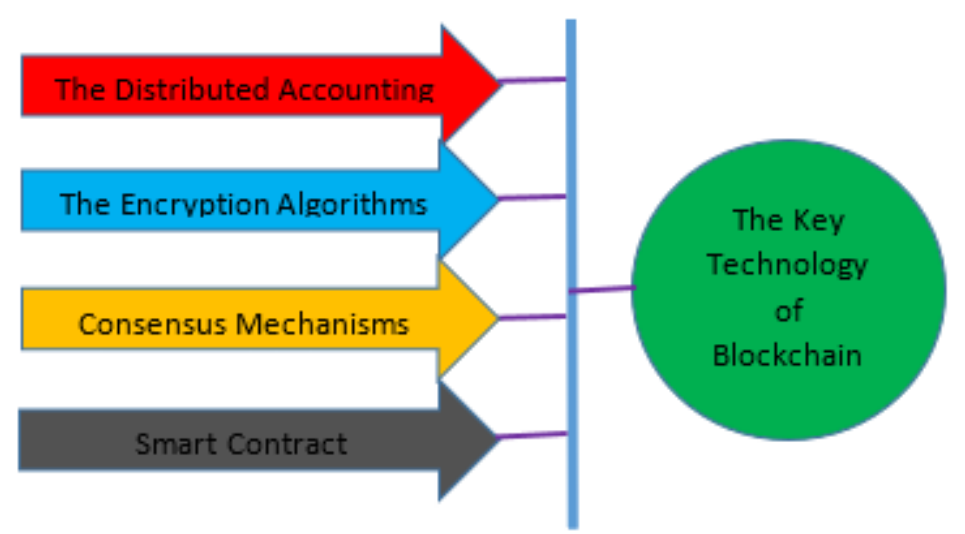

Figure 1. The four key technology of Blockchain

\section{Distributed Accounting}

This technology allows every participant to have a copy of all the transactions in the blockchain for complete transparency. Unlike the traditional accounting where there is a central authority, the distributed accounting enable all nodes in the chain to keep records of all other nodes and thus, making it extremely difficult for hackers to hack into blockchain system as they would have to change every block across all the distributed version of the chain [18].

\section{Encryption Algorithm}

Blockchain adopts two cryptographic encryption algorithms (asymmetric encryption and hash function) in order to ensure security of information. The asymmetric algorithm consists of two key: private key and public key. The private key is used for encryption at the sender's end while the receiver's side used the public key for decryption [19]. More so, blockchain uses hash function to make the information from the previous block not easily alterable. The hash function is a cryptographic algorithm that is not easily reversible [11]. It has the capability of converting any kind of data into character strings. Thus, providing security through encryption [18].

\section{Consensus Mechanism}

This is the fault-tolerant mechanism use to achieve necessary agreement in a blockchain system. It helps to validate every block before it is added to the chain (every participating node in the network must attest to the validity of a block before it is added). Consensus mechanism helps to identify legitimate blocks and prevent tampering by using proofs. These proofs (proof of work, proof of stake, proof of authority, Proof of Capacity etc.) discourages frauds. In the bitcoin network, consensus is reached through the proof of work [10],[18]. Forging a non-existing record in the blockchain system is extremely difficult and as such eliminating the possibility of counterfeiting except if the attacker has more computing capability (at least 51\%) than the network of miners to be able to fool the network successfully. However, this is impractical, where many nodes join the chain [18].

\section{Smart Contract}

The smart contract is a digital agreement and self-executing transaction protocol stored on the blockchain system. The essence of the smart contract is to provide superior security, reduce cost and eliminate delay that are inherent in the traditional contract laws. Smart contracts digitally facilitate, verify or enforce a credible negotiation of a contract without a third party. Smart contracts are stored on the public database of the blockchain and cannot be changed by an individual [18].

\section{THE KEY CHARACTERISTICS OF BLOCKCHAIN}

\section{Decentralization}

Transactions on blockchain are not stored in a centralized database where all transactions need to be validated by a central authority which results to performance bottlenecks at the central server. Blockchain technology adopts the concept of P2P networking paradigm. The operation of P2P is based on decentralized database system where all nodes have equal right and obligation. There is no central authority in a decentralized transaction process, therefore, the performance bottlenecks at the central server is eliminated in a decentralized system [10].

\section{Openness}

The public or permissionless blockchain are open to everyone. Anyone can join or leave at any time without the need for a third-party approval. Mutual trusts are not needed for data exchange between nodes, the rules of operation are open and transparent to all nodes. Blockchain uses trusted mathematical algorithms to regulate the activities of information in the chain [19]. For any update in any node, mutual authentication of other nodes is required. Therefore, the openness characteristics of blockchain ensures public verifiability [20]. 


\section{Autonomy}

Operating rules and protocols in blockchain are based on consensus and cannot be influence by any single node or central authority. Any node in the chain can read, write and verify blocks at any time. Since, there is no coordinating node, blocks are jointly maintained by all nodes in the network [20].

\section{Persistency}

Transactions are permanently stored in the blockchain once it has been verified and added to the chain of transactions. Modification are only possible if a node has a computing capability of at least $51 \%$ else, modification is null and void. However, this is practically impossible where more nodes join the chain of transaction. Persistency guarantees high stability and reliability data in the chain [20].

\section{Anonymity}

In blockchain system, data exchange between nodes are govern by consensus, hence, parties in the chain need not disclose their identities. Since there is no central authority that keeps users' information privately, the rules of the program in the blockchain are used to give trust [20].

\section{APPLICATIONS OF BLOCKCHAIN}

Some areas where blockchain plays a great role are provided in this section. Figure 2 depicts some application domains of blockchain.

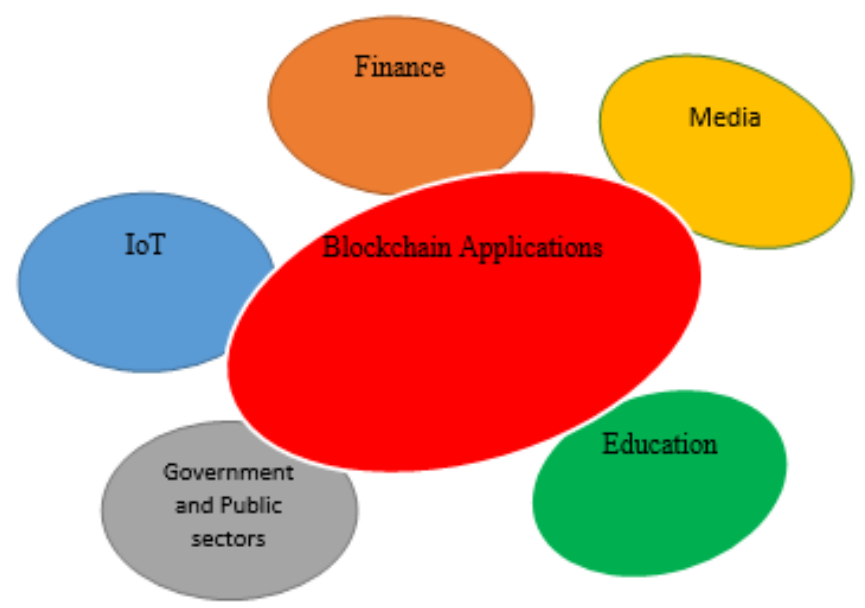

Figure 2. Some application domains of blockchain

\section{Finance}

The emergence of blockchain technology has brought a huge success in the field of finance as it ensures high trust and robustness of transactions between permissioned parties without a central authority. Blockchain is steadily reshaping the transaction process by allowing the inflow of liquid cash through intelligent contracts and also prompting cross border payments in real time [20]. Blockchain also finds application in clearing and settlement of financial assets such as bonds, stocks or derivatives by transcripting assets into blocks; thereafter, it is validated by parties involved for legitimacy which makes it easier to detect any form of fraudulent activities. Apart from revolutionizing the traditional finance activities, contemporary forms of finance such as crowdfunding can also be improved with the blockchain technology [20].

Media

The media industry poses a lot of challenges which includes the piracy of intellectual properties, royalty payment and data piracy [21]. These challenges are due to the widespread sharing of media contents that infringe on copyright properties. Blockchain have the capability to revolutionized the media industry by using the shared ledger approach to address the data right, piracy and payment challenges that are inherent in the media industry. Multiple existence of digital assets in various location can also be prevented with blockchain technology. The distributed ledger system also allows files to be shared and distributed while maintaining data integrity. Thus, making piracy virtually impossible [21].

Internet of Things (IoT)

The network of physical things (smart object) that are incorporated with sensors, software and other technologies such as Radio Frequency Identifier (RFID), cloud/fog computing, wireless communication and so on to allow data exchange and connections with other devices over the internet is known as IoT [22]. The applications of IoT are numerous, however, there are some security concerns regarding IoT that need urgent attention. The proliferation in IoT products give internet 
fraudsters a higher chance of infringing data integrity. Incorporating IoT and blockchain guarantees a higher level of security and prevent tampering by utilizing shared ledger technology to keep things smart. Parties can also share and access IoT data without any central control authority [22]. Transactions can be verified to prevent dispute and build trust among all permissioned network members.

\section{Government and Public Sectors}

Government and public sector still remain the most corruptible sector around the globe. The various types of corruption in this sector includes; bribery, embezzlement, abuse of office (favoritism and nepotism), fund siphoning etc. A digital government based on blockchain can eliminate any form of corruption while simultaneously building trust and accountability [23]. The distributed ledger system also eliminates single point of control; thus, resources can be shared between participants in a more transparent mode. Blockchain technology can also be deployed to support other governmental activities such as supply chain traceability, digital management, voting, healthcare corporate registration and management of legal entities.

\section{Education}

Blockchain can be deployed to educational institutions to enhance the security and efficiency of the institutions. The distributed ledger system has the potential to ensure that the identity, privacy and security of student's data is not compromised by ensuring immutability through its hash chain. For example, it is practically impossible for a student to make an alteration to his past record stored on blockchain but is much easier to do that with the paper mode [24]. In addition, privacy is ensured through blockchain; it is not about storage of the data but rather a hash of the data. Through blockchain, student records become public and easier to share with employers and universities for further personal development opportunities.

\section{BLOCKCHAIN CHALLENGES}

Blockchain as an emerging technology is facing numerous challenges which include the following:

\section{Scalability}

Due to the increase in transactions on a daily basis, the blockchain becomes heavy, especially the public blockchain. For instance, the bitcoin blockchain has exceeded 300 gigabytes in size and keeps growing at 1 MB per block every ten minutes while the Ethereum archive nodes now takes up to 4 terabytes of space [25]. Also, the bitcoin blockchain processing capability is limited to not more than 7 transactions per second, simply because of the restriction of block size and the time frame that is required to generate new block. Since the processing capacity is small, transactions of lower priority may experience delay because some miners work on transactions in order of priority. Thus, large block size would lower down the propagation speed and could also amount to blockchain branches. Therefore, scalability problem is inherent in blockchain. However, there are ongoing researches which are focused on addressing the scalability problem.

\section{Lack of Privacy}

Lack of privacy is another challenge with the technology of blockchain [25]. Though, it is generally believed that blockchain is very safe as users only make transaction with the generated address rather than revealing self-identity. In the case of information leakage, user could also generate many addresses [25]. However, [26] revealed that transactions privacy's are not guaranteed since the values of all transactions and balances for each public key are not hidden. Also, [27] shows that a user's bitcoin transactions can be linked to reveal user's profile. A method capable of linking user's pseudonym to IP address even when users are behind firewall or NAT was also proposed by [27].

\section{Lack of Talent}

There is lack of talent in building decentralized applications. It takes time to educate employees on how to work with blockchain. Blockchain is not yet taught at many educational institutes. Only about $50 \%$ of the world top institutions offer a course on blockchain technology. Therefore, it is important that organizations and academia need to work together to ensure all the latest technologies are introduced into the curriculum [28].

\section{Power consumption}

The level of power consumption due to the nature of the decentralized network is another great concern of the distributed digital ledger technology. The proof of work consensus mechanism still remains the mostly widely used consensus mechanism. It solves complicated puzzles which uses large amount of energy to complete the task. It is estimated that it consumes not less than 65 terawatt-hour per year [29].

\section{Resilience and Irreversibility}

Another challenge that is encountered in the blockchain is resilience and irreversibility. Once data has been entered and appended by the network, it cannot be reversed. Thus, reliability and accuracy of data entry cannot be guaranteed by the system [30]. For instance, if sets of bad data are correctly entered, they will end up on blockchain; similarly, if a document containing false information is entered correctly into the blockchain, it remains in the transaction chain. 


\section{Selfish Mining}

It is documented in literatures that nodes with at least $51 \%$ computing capability have the potential to control the system. However, [25] revealed a node with less than that can still be perilous. Bitcoin mining relies on miners who solve complex mathematical puzzles to generate coins and the protocol is configured to reward miners base on their output. In selfish mining strategy, groups of miners joined to increase their revenue by keeping newly generated (mined) blocks from the main blockchain and hence creating a split and only make it public with systems within their private network or make it available to the public when some requirement are reached. Miners will have to admit the private branch when is longer than the current public chain. According to [25] "Once a selfish mining pool reaches a threshold (of a public blockchain), rational miners would be attracted to join the selfish miners to reap higher revenue as compared to other pool".

\section{CONCLUSION}

Blockchain is one of the most rapidly emerging technologies. It shows a promising light in the evolving digital revolution, especially in industries, businesses, and governance as well as in other areas in which improved operational transparency, auditability, accountability, cost effectiveness and efficiency are non-negotiable. Having examined the various building blocks of the blockchain technology and its inherently extensive potentials, it was observed that the technology is interestingly a technology of the future and its adoption in the various sectors of any economy including government, business, education, etc. appears to offer the desired immunity to breaches of trust, fraud, data theft, to mention a few, in the cyberspace. With the cyberspace emerging the global socioeconomic hub owing to its great offerings albeit the increasing spate of security challenges, the blockchain technology is an attractive option though not without its own challenges. Nevertheless, it is submitted that its benefits far outweighs its challenges and that these challenges are amenable to advancement in computing technology, awareness programs, and use of fair benchmarks. Notably, higher computing power and scalability are presently being addressed by a plethora of computer hardware manufacturers including Oracle, IBM, and Huawei using quantum computing and other distributed and parallel computing architectures [30]. In the last decade there has been a growing awareness on environmental sustainability and green computing practices are being adopted by computer hardware manufacturers to reduce the power consumption demands of computing equipment and some global policies and programs have also been implemented across several jurisdictions[31-33]. In respect of selfish mining, it is suggested that the design and adoption of fair benchmarked algorithms would curtail this problem whereas irreversibility and resilience are all connected to modifying the structure of the blockchain system to accommodate extensive data validation checks prior to acceptance and replication to other nodes. Since, irreversibility is to a large extent connected to the blockchain software and embedded algorithms it is therefore imperative to review the present business logic associated with such algorithms. In conclusion, it is submitted that the blockchain technology is considered the foundation on which the future technology security apparatus in the cyberspace would be based and once the identified problems are addressed this evolving technology would help the socioeconomic and political ecosystem to thrive with less burden of budgets, legislation, and machineries for prevention and control of cybercrimes.

\section{REFERENCES}

[1] F. Casino, T. K Dasaklis, and C. Patsakis "A systematic literature review of blockchain-based applications: Current status, classification and open issues". Telematics and Informatics, 2019,. 36, pp.55-81.

[2] A. Collomb and K. Sok "Blockchain/distributed ledger technology (DLT): What impact on the financial sector?" Digiworld Economic Journal, 2016,. (103).

[3] X. Pan, X. Pan, M. Song, B. Ai, and Y. Ming, "Blockchain technology and enterprise operational capabilities: An empirical test". International Journal of Information Management, 2020, 52, p.101946

[4] F. Dai, Y. Shi, N. Meng, L. Wei, and Z. Ye "From Bitcoin to cybersecurity: A comparative study of blockchain application and security issues". In 2017 4th International Conference on Systems and Informatics (ICSAI) November 2017,. (pp. 975979). IEEE.

[5] P. W. Abreu, M. Aparicio, and C. J Costa "Blockchain technology in the auditing environment". In 2018 13th Iberian Conference on Information Systems and Technologies (CISTI) June 2018,. (pp. 1-6). IEEE.

[6] J. L. Zhao, S. Fan and J. Yan "Overview of business innovations and research opportunities and blockchain and introduction to the special issue (2016)": 1-7

[7] I. Riabi, H. K. B Ayed, and L. A. Saidane "A survey on Blockchain based access control for internet of Things. In 2019 $15^{\text {th }}$ International Wireless Communications \& Mobile Computing Conference(IWCMC) June 2019,. (pp. 502-507) IEEE

[8] G. Zyskind, O. Nathan, and A. Pentland. Enigma: Decentralized computation platform with guaranteed Privacy (2015),. arXiv preprint arXiv:1506.03471

[9] G. Paul,P. Sarkar, and S. Mukherjee. "Towards a more democratic mining in bitcoins". In International Conference on Information Systems Security. December 2014,. (pp. 185-203). Springer, Cham.

[10] J. Li, and X. Wang "Research on the application of blockchain in the traceability system of agricultural products". In 2018 2nd IEEE Advanced Information Management, Communicates, Electronic and Automation Control Conference (IMCEC) May 2018,. (pp. 2637-2640). IEEE.

[11] R. C. Global "Blockchain for Traceability in Minerals and Metals Supply Chains: Opportunities and Challenges" Self published. 2017,.1(1): p1-21. 
[12] D. Aliu, S. M. Shaba, M. O. Momoh, P. U Chinedu and W. Nwankwo "A Computer Security System for Cloud Computing Based on Encryption Technique" Computer Engineering and Applications 10(1), February 2021,. (pp. 41-54).

[13] S. Underwoo. Beyond Bitcoin. Commun. ACM 59, October 2016,. (15-17)

[14] I. K. Lim, Y. H. Kim, J. G. Lee, J. P Lee, H. Nam-Gung and J. K. Lee. "The Analysis and Countermeasure on Security breaches of Bitcoin”. In International Conference on Computational Science and Its Applications. Springer International publishing 2014,. (pp. 720-732)

[15] I. Eyal and E. G. Sirer, "Majority is not enough: Bitcoin mining is vulnerable," In Financial Cryptography and Data Security: 18th International Conference. Springer Berlin Heidelberg, 2014,. pp. 436- 454.

[16] A. Sapirshtein, Y. Sompolinsky, and A. Zohar, "Optimal selfish mining strategies in bitcoin," In Financial Cryptography and Data Security: 20th International Conference, FC 2016, Christ Church, Barbados,February 22-26, 2016. Springer Berlin Heidelberg, 2017,. pp. 515-532.

[17] K. Nayak, S. Kumar, A. Miller, and E. Shi, "Stubborn mining:Generalizing selfish mining and combining with an eclipse attack," In 2016 IEEE European Symposium on Security and Privacy (EuroS P), 2016, pp. 305-320.

[18] P. T. Duy, D. T. T. Hien, D. H. Hien, and V. H. Pham "A survey on opportunities and challenges of Blockchain technology adoption for revolutionary innovation". In Proceedings of the Ninth International Symposium on Information and Communication Technology December 2018,. (pp. 200-207).

[19] X. M. Yang, X. Li, H. Q. Wu, and K. Y. Zhao. "The application model and challenges of blockchain technology in education”. Modern distance education research 2017,. 2, pp.34-45.

[20] A. Muneeza, N. A. Arshad, and A. T Arifin "The application of blockchain technology in crowdfunding: towards financial inclusion via technology". International journal of management and applied research, 5(2), 2018,. pp.82-98.

[21] T. Dogru, M. Mody, and C. Leonardi "Blockchain technology \& its implications for the Hospitality Industry". Boston University 2018.

[22] E. M. Dogo, A. F. Salami, N. I. Nwulu, and C. O. Aigbavboa. Blockchain and internet of things- based technologies for intelligent water management system. In Artificial intelligence in IoT 2019,. (pp. 129-150). Springer, Cham.

[23] A. I. Sanka, and R.C. Cheung "Blockchain: Panacea for Corrupt Practices in Developing Countries". In 2019 2nd International Conference of the IEEE Nigeria Computer Chapter (NigeriaComputConf) October 2019,. (pp. 1-7). IEEE.

[23] R. Neisse, J. L. Hernández-Ramos, S. N. Matheu, G. Baldini, and A. Skarmeta, "Toward a blockchain-based platform to manage cybersecurity certification of IoT devices". In 2019 IEEE Conference on Standards for Communications and Networking (CSCN) ) October 2019,. (pp. 1-6). IEEE

[24] A. I. Sankaa, M. Irfana, Huangb, I. and R. C. Cheunga. "A Survey of Breakthrough in Blockchain Technology: Adoptions, Applications, Challenges and Future Research". Telematics and Informatics 36, 2019,. p55-81.

[25] Z. Zheng, S. Xie, H. N. Dai, X. Chen and H. Wang, "Blockchain Challenges and Opportunities: A Survey". International Journal of Web and Grid Services, 2018,. 14 (4), pp 352-375

[26] J. Barcelo, (2014) User Privacy in the Public Bitcoin Blockchain.

[27] A. Biryukov, D. Khovratovich, and I. Pustogarov. (2014) "Deanonymisation of clients in bitcoin p2p network", Proceedings of the 2014 ACM SIGSAC Conference on Computer and Communications Security, New York, NY, USA, pp.15-29

[28] X. Pan, X. Pan, M. Song, B. Ai, and Y. Ming. "Blockchain technology and enterprise operational capabilities: An empirical test”. International Journal of Information Management, 2020, 52 (3), p.101946

[29] J. Wu and N. K. Tran. Application of blockchain technology in sustainable energy systems: An overview. Sustainability, October 2018. 10(9), p1-22

[30] K.A. Britt,and T.S. Humble."High-Performance Computing with Quantum Processing Units". ACM J. Emerg. Technol. Comput. Syst.,2017,. 1, 1, Article 1, PP.1-13.

[31] W. Nwankwo, A.S. Olayinka, K.E. Ukhurebor. "Green Computing Policies and Regulations: A Necessity?". International Journal of Scientific and Technology Research, 2020,. 9(1).

[32] W. Nwankwo, and K.E. Ukhurebor. "Data Centres: A Prescriptive Model for Green and Eco-Friendly Environment in the Cement Industry in Nigeria". International Journal of Scientific and Technology Research, 2020,. 9(5), pp. 239-244

[33] W. Nwankwo, and U.P. Chinedu."Green Computing: A Machinery for Sustainable Development in the Post-Covid Era". In Albert Sabban(Ed.), Green Technologies and Computing Industry. IntechOpen, United Kingdom, 2020. 